TERMINAL CONTEXT IN

CONTEXT-SENSITIVE GRAMMARS ${ }^{\dagger}$

by

Ronald V. Book

$10-71$

Center for Research in Computing Technology 
TERMINAL CONTEXT IN

CONTEXT-SENSTTIVE GRAMMARS ${ }^{\dagger}$

by

Ronald V. Book

$10-71$

\author{
Center for Research in Computing Technology \\ Harvard University \\ Cambridge, Massachusetts 02138
}

\footnotetext{
This research was supported in part by the National Aerom nautics and Space Administration under Grant No. NGR-22-007-176 and by the National Science Foundation under Grant No. GJ-803. Part of this research was performed while the author was visiting the Department of System Science, University of California, Los Angeles.
} 


\title{
Terminal Context in Context-Sensitive Grammars
}

by

Ronald V. Book

\begin{abstract}
If every non-context-free rule of a context-sensitive (with erasing) grammar has as left context a string of terminal symbols and the left context is at least as long as the right context, then the language generated is context-free. If every non-context-free rule of a contextsensitive (with erasing) grammar has as left and right context strings of terminal symbols, then the language generated is context-free.
\end{abstract}


Introduction.

It is well-known that the family of context-sensitive grammars generates languages which are not context-free and that it is undecidable whether a context-sensitive grammar generates a context-free language. However the mechanism by which the use of context allows a non-context-free language to be generated is not well understood (in fact, the question itself is vague: what does context do for you?). In this paper it is shown that when certain nontrivial constraints are placed on the form of the rules of a context-sensitive (with erasing) grammar only context-free languages will be generated. These constraints involve the use of terminal strings as part of context. The first restriction is that for every non-context-free rule, the left context is a string of terminal symbols which is at least as long as the (arbitracy) right context. (It is shown that the length restriction cannot be weakened.) The second restriction is that both left and right context be strings of terminal symbols.

If one is constructing a context-sensitive gramar to generate some non-context-free language, then one often proceeds as if context can be used to "store and transmit" information. Thus one builds rules so that "messages" or "pulses" are transmitted along a string in the course of the derivation. Sometimes this effect is achieved by building a gramar which imitates the action of a Turing machine; hence, the action of the 
read-write head must be imitated as it travels back and forth across the tape.

The "ability to send messages" has not been formalized in such a way as to explain "what context does for you," although some properties of the structure of derivations have been studied $[1,5,6]$. However this notion does provide an intuitive "handle" for studying some questions and for gaining perspective on some results on context-sensitive gramars and languages. I The results established in this paper may be interpreted as constraining the "message-sending" capacity by means of strings of terminal symbols which act as "barriers" when used as context.

There are two somewhat related results in the literature. Hibbard [7] has shown that if $G=(V, \Sigma, R, X)$ is a grammar and $<$ as a partial order on $V$ with the property that for every rule $Z_{1} \ldots Z_{p} \rightarrow Y_{1} \ldots Y_{q}$

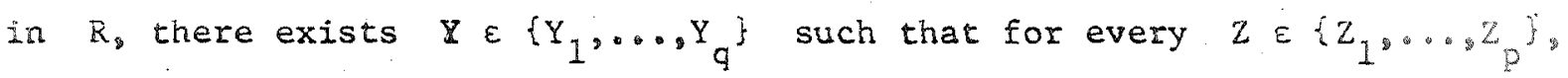
$Z<Y$, then $L(G)$ is context-free. Ginsburg and Greibach [4] have shown that if $G=(V, E, R, X)$ is a grammar such that every rule in $R$ is of the form $\rho \rightarrow \theta$ where $\rho \varepsilon(V-\Sigma)^{*}$ and $\theta \varepsilon V^{*} \Sigma V^{*}$, then $L(G)$ is contextfree. Both of these results may be interpreted as contraining the "messagesending" capacity by erecting "barriers." Neither result appears to imply or be implied by the results established here.

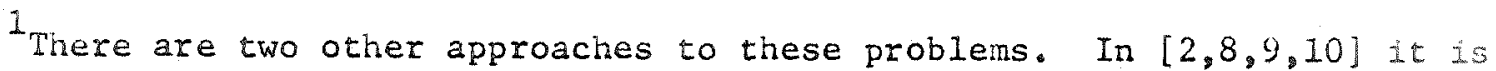
shown that for an arbitrary grammar certain types of derivations yield only context-free languages. Also, one can consider "regulating" the application of rewriting rules, such as in matrix grammars, programmed grammars, etc. See [II] for a summary of results in this area.
} 
Section 1.

For the most part the notation used in this paper is that of [3] and the reader is referred to [3] for facts about context-free languages, regular sets, and gsm mappings. However there are certain conventions which need to be emphasized here.

A grammar is quadruple $G=(V, \Sigma, R, X)$ where $V$ is a finite set of symbols, $\Sigma \subset V$ is the set of terminal symbols, $X \in V-\Sigma$, and $R$ is a finite set of rewriting rules (productions) of the form $\alpha_{1} y_{1} \ldots \alpha_{n} y_{n} \alpha_{n+1} \rightarrow$ $\alpha_{1} w_{1} \ldots \alpha_{n} w_{n}^{\alpha}{ }_{n+1}$, with each $\alpha_{i} \varepsilon \Sigma^{*}, y_{i} \varepsilon(V-\Sigma)^{*}(V-\Sigma), w_{1} \varepsilon V^{*}$, and for some $i, w_{i} \neq y_{I}{ }^{2}$ If $p \rightarrow \theta \in R$, then for any $\alpha, \beta \in V^{*}$, write $\alpha \rho \beta \Rightarrow \alpha \Theta \beta$ and say that the rule $\rho \rightarrow \theta$ is applicable to the string $\alpha \rho \beta$ and that $\rho \rightarrow \theta$ transforms $\alpha \rho \beta$. A derivation in $G$ is a sequence $\Gamma_{0}, \Gamma_{1}, \ldots, \Gamma_{n} \varepsilon V^{*}$ such that for $i=1, \ldots, n, \Gamma_{i-1} \Rightarrow \Gamma_{i}$. The transitive reflexive closure of $\rightarrow$ is $\stackrel{*}{\rightarrow}$. The language generated by $G$ is $L(G)=\left\{w \in \Sigma^{*} \mid X \stackrel{*}{\Rightarrow} w\right\}$. If $G=(V, \Sigma, R, X)$ is a grammat and $\Gamma_{0} \Rightarrow \Gamma_{1} \Rightarrow \ldots \Rightarrow \Gamma_{n}$ is a derivation in $G$, then a production sequence [5] for this derivation is a sequence of $n$ ordered pairs, $\left\{\left\langle\left(B_{i}, P_{i}, C_{i}\right),\left(B_{i}, Q_{i}, C_{i}\right)\right\rangle\right\}_{i=1}^{n}$ where for each $i=1, \ldots, \Omega_{2}, B_{i} P_{i} C_{i}=\Gamma_{i-1}, B_{i} Q_{i} C_{i}=\Gamma_{i}$ and $P_{i} \rightarrow Q_{i} \varepsilon R_{0}$

A gramar $G=(V, \Sigma, R, X)$ is Type $O$ (or context-sensitive with erasing) if each rule in $R$ is of the form $\alpha Z \beta \rightarrow \alpha \gamma \beta, \alpha, \beta, \gamma \varepsilon V^{*}, Z \varepsilon V=\Sigma$.

2 For any set $T$ of symbols, $T^{*}$ is the free semigroup with identity generated by $T$. 
It is well-known that a set $L$ is recursively enumerable if and only if there is a Type 0 gramar $G$ such that $L(G)=L$.

A grammar $G=(V, \Sigma, R, X)$ is context-free if each rule in $R$ is of the form $Z \rightarrow \gamma, Z \in V-\Sigma, \gamma \varepsilon V^{*}$. (In any gramar a rule of this form is called a context-free rule.) A language $L$ is context-free if and only if there is a context-free grammar $G$ such that $L(G)=L$.

The length of a string $w$ is denoted by $|w|$. 
Section 2.

In this section we establish Theorem $I$ below and show that the hypothesis cannot be weakened.

Theorem 1. Let $G=(V, \Sigma, R, X)$ be a Type $O$ grammar. If each non-contextfree rule in $R$ is of the form $\alpha Z \beta \rightarrow \alpha \gamma \beta$ where $\alpha \varepsilon \Sigma^{*}, z \varepsilon V \ldots \Sigma$, $B, \gamma \in V^{*}$, and $|\alpha| \geq|\beta|$, then $L(G)$ is context-free.

A non-context-free rule in $R$ has left context which is a terminal string and right context which is no longer than the left. (no other restriction is placed on the right context). These two restrictions imply that a "message" cannot be "transmitted" to the left over a string of symbols longer than $1+m(m+1) / 2$ where $m=\max \{|\alpha| \mid \alpha Z \beta \rightarrow \alpha \gamma \beta \varepsilon R\}$. Thus any $w \in L(G)$ can be generated by a derivation such that at each step the transformed symbol is no farther than $m(m+1) / 2$ from the leftmost nonterminal syabol in the string being transformed-hence, $L(G)$ is concextfree. In order to prove this, we first state some definitions and review some facts about grammars and languages.

Let $G=(V, \Sigma, R, X)$ be a gramar. For $\Gamma, \psi \varepsilon V^{*}, \tau \geq 1$, if $I \Rightarrow \psi$ where $\Gamma=\alpha Z \ldots Y B, Z$ is the leftmost nonterminal symbol in $\Gamma$ (i.e., $\alpha \in \Sigma^{*}$ and $\left.Z \in V-\Sigma\right), Y$ is the transformed symbol in $\Gamma \Rightarrow \Psi$, and $|Z \ldots Y| \leq t$, then $\Gamma \Rightarrow \Psi$ is $t$ bounded. If $\Gamma \Rightarrow \Psi$ is $t$ bounded, then it is $r$ bounded for every $r \geq t$. A derivation is a t-bounded derivation if each step is $t$ bounded. For any $t \geq 1$ ZEFT $(\tau, G)=$ 
$\left\{w \in \Sigma^{*} \mid\right.$ there is a t-bounded derivation $X \Rightarrow \ldots \Rightarrow w$ in $\left.G\right\}$. Clearly, for every $t \geq 1, \operatorname{LEFT}(t, G) \subseteq L(G)$. In $[2,8]$ it is shown that for any grammar $G$ and any $t \geq 1, \operatorname{LEFT}(t, G)$ is a context-free language.

For any $t \geq 1$, let $M(t)=t(t+1) / 2$. For a Type o grammar $G=(V, \Sigma, R, X)$, let $m=\max \{|\alpha| \mid \alpha Z \beta \rightarrow \alpha \gamma \beta \varepsilon R\}$ and let $M_{G}=1+M(m)$. If $G$ satisties the hypothesis of Theorem 1 , then for any rule $\alpha Z \beta \rightarrow \alpha \gamma \beta \varepsilon R_{8}$ $|\beta| \leq M(|\beta|)<M_{G}$ since $|\alpha| \geq|\beta|$. To prove Theorem 1 we shaII show that $\operatorname{LEFT}\left(M_{G}, G\right)=L(G)$ so that $L(G)$ is context-free because $\operatorname{LEFT}\left(M_{G}, G\right)$ is context-rree.

The formal argument proving Theorem 1 is based on the following observation:

(i) Consider $\mu \alpha Z x_{1} \ldots x_{n}$ where $\mu_{0} \alpha \varepsilon \Sigma^{*}$. Suppose one wishes to apply the rule $\alpha Z \beta \rightarrow \alpha \gamma \beta$ in order to transform 2 . Consider $x_{1} \ldots x_{n}$. If $B$ is a prefix of $x_{1} \ldots x_{n}$, then this rule can be applied. If not, then other rules must be applied to $x_{1} \ldots x_{2}$ in order to obtain a string with $B$ as prefix. 
(ii) Suppose that $\beta=x_{1} \ldots x_{q}$ where $q<n$ and that some rule must be applied to $x_{1} \ldots x_{n}$ in order to transform $x_{c+1}$ so that eventualiy $B$ is obtained as a prefix. Further, suppose that the rule to be applied is a context-sensitive rule, so that its left context is a suffix of $x_{1} \ldots x_{q}$ (since $z \in V-\Sigma_{\text {g }}$ $z$ cannot occur as part of terminal left context). Thus the rule to be applied must have left context which is shorter than $q+1 \leq|\beta|$. But $|\alpha| \geq|\beta|$ so that the left context of this rule is shorter than $\alpha$.

(ii) By induction, it is seen that if it is possible to apply rules to $x_{1} \ldots x_{n}$ in order to obtain a string with $\beta$ as prefix, then it is possible to do this by transforming symbols that are no farther than $1+2+\ldots+|\alpha|$ from $z$, so that each step in the resulting derivation is $M(|\alpha|)+1$ bounded. Since $M_{G} \geq I+M(|\alpha|)$, the resulting derivation is $M_{G}$ bounded.

The result of these observations is argued formally through two lemmas (the proof of Lemma I containing the main argument). The theorem then follows easily. 
Lemma 1. Let $G=(V, \Sigma, R, X)$ be a Type 0 grammar which satisfies the hypothesis of Theorem 1 . For any $\beta \varepsilon V^{*}$ and any $n \geq 1$, if $\Gamma_{0} \Rightarrow \ldots \Rightarrow \Gamma_{n}$ is a derivation of length $n$ in $G$ such that $\beta$ is not a prefix of $\Gamma_{0}$ but is a prefix of $r_{n}$, then there exist $\pi_{0} \ldots, \pi_{n} \in V^{*}$ such that $\pi_{0}=\Gamma_{0}, \Pi_{n}=\Gamma_{n}, \pi_{0} \Rightarrow \ldots \Rightarrow \pi_{n}$ is a derivation of length $n$ in $G$, and the step $\pi_{0} \Rightarrow \pi_{1}$ is $M(|B|)$ bounded. Proos. Since $B$ is not a prefix of $\Gamma_{0}, \beta \neq$ e. The proof proceeds by induction on $|\beta|$.

(i) For any $n \geq 1$, let $\Gamma_{0} \Rightarrow \ldots \Rightarrow \Gamma_{n}$ be a derivation of length $n$ in $G$, let $\left\{\left\langle\left(B_{i}, P_{i}, C_{i}\right),\left(B_{i}, Q_{i}, C_{i}\right)>\right\}_{i=1}^{n}\right.$ be a production sequence for this derivation, and let $\Gamma_{0}=Y_{1} \ldots Y_{t}, t \geq I_{1}$ each $Y_{j} \varepsilon V_{0}$ If $|\beta|=1$, then $\beta \varepsilon V$. Since $\beta$ is not a prefix of $r_{0}=Y_{1} \ldots Y_{t}, \beta \neq Y_{1}$. But $\beta$ is a prefix of $r_{n}$ so that there is some step in $\Gamma_{0} \Rightarrow \ldots \Longrightarrow \Gamma_{n}$ which transforms $Y_{1}$. Hence, $Y_{I} \in V-\Sigma$. Thus $Y_{1}$ does not occur as part of the left context of any rule in $R$ (since such context is in $\left.\Sigma^{*}\right)$. Let $\Gamma_{k-1} \Rightarrow \Gamma_{k}$ be the first step which transforms $\Psi_{I^{\prime}}$ Since $Y_{1}$ is the leftmost symbol in $\Gamma_{0}$ and since $\Gamma_{k-1} \rightarrow r_{k}$ is the first step which transforms $Y_{1}$, for each $i=1, \ldots, k$ $Y_{1}$ is the leftmost symbol of $\Gamma_{i-1}=B_{i} P_{i} C_{i}$. Thus $Y_{1}$ cannot be used as part of right context for any of the rules $P_{i} \rightarrow P_{1}$, $1 \leq i \leq k-1$. Hence for each $i=1, \ldots, k-1, \vec{B}_{i}=Y_{1} D_{i}$ for some $D_{i} \in V^{*}, B_{k}=e$, and $P_{k} \rightarrow Q_{k}$ is a context-free rule 
with $P_{k}=Y_{1}$. Thus the derivation $\Pi_{0} \Rightarrow \ldots \Rightarrow \Pi_{n}$ can be constructed by first applying $P_{k} \rightarrow Q_{k}$ to $Y_{1}$ in $\Gamma_{0}$, then imitating the derivations $\Gamma_{0} \Rightarrow \ldots \Rightarrow \Gamma_{k-1}$ and $\Gamma_{k} \Rightarrow \ldots \Rightarrow \Gamma_{n}$ Since $Y_{1}$ is transformed by $\Pi_{0} \Rightarrow \Pi_{1}$, this step is $M(|B|)=1$ bounded. The construction of the derivation $\pi_{0} \Rightarrow \ldots \Rightarrow \pi_{n}$ is carried out below.

$$
\text { Let } D_{k}=\text { e. Thus } \Gamma_{k-1}=B_{k-1} Q_{k-1} C_{k-1}=Y_{1} D_{k-1} Q_{k-1} C_{k-1}
$$

and $\Gamma_{k-1}=B_{k} P_{k} C_{k}=Y_{1} C_{k}$ so that $C_{k}=D_{k-1} Q_{k-1} C_{k-1}$. Construct $\Pi_{0} \ldots, \pi_{n}$ as follows. Let $B_{i}^{\prime}=e, P_{1}^{p}=y_{1}=P_{k}, Q_{1}^{\prime}=Q_{k}$ and $C_{1}^{t}=Y_{2} \ldots Y_{t}=D_{1} P_{1} C_{1}$; let $\Pi_{0}=B_{1}^{i} P_{1}^{i} C_{1}^{i}=P_{k} D_{1} P_{1} C_{1}$ and $\Pi_{1}=B_{1}^{\prime} Q_{1}^{\prime} C_{1}^{\prime}=Q_{k} D_{1} P_{1} C_{1}$ so that $\Pi_{0}=Y_{1} \ldots Y_{t}=\Gamma_{0}$ and $\Pi_{0} \Rightarrow \Pi_{I}$. For $j=2, \ldots, k$, let $B_{j}^{n}=Q_{k} D_{j-1}, P_{j}=P_{j=1}$ $Q_{j}^{q}=Q_{j-1}, \quad C_{j}^{q}=C_{j-1}$ and $\Pi_{j}=B_{j}^{q} Q_{j}^{q} C_{j}^{q}$. Then $B_{2}^{8} P_{2}^{1} C_{2}^{1}=$ $Q_{k} D_{1} P_{I} C_{I}=\Pi_{1}$, and for $j=2, \ldots, k, \Pi_{j}=B_{j}^{i} Q_{j}^{i} C_{j}^{p}=Q_{k} D_{j-1} Q_{j-1} C_{j-I}=$ $Q_{k} D_{j} P_{j} C_{j}=B_{j+1}^{\prime} P_{j+1}^{0} C_{j+1}^{0}$ (since $\Gamma_{j-1}=B_{j-1} Q_{j-1} C_{j-1}=$ $\left.Y_{1} D_{j-1} Q_{j-1} C_{j-1}=B_{j} P_{j} C_{j}\right)$. Thus $\pi_{k}=Q_{k} D_{k-1} Q_{k-1} C_{k-1}=Q_{k} C_{k}=$ $\Gamma_{k^{*}}$ For $i=k+1, \ldots, n_{\text {, }}$ let $\Pi_{i}=\Gamma_{i .}$. Thus $\Pi_{0} \Rightarrow \ldots \Rightarrow \pi_{k}=r_{k} \Rightarrow \ldots \Rightarrow \Pi_{n}=\Gamma_{n}$ is a derivation of length $n$ in $G_{0} \Pi_{0}=\Gamma_{0}, \Pi_{n}=\Gamma_{n}$, and the step $\Pi_{0} \Rightarrow \Pi_{1}$ is $M(|B|)=1$ bounded.

(ii) Assume the result for all $\beta \varepsilon V^{*}$ such that $|\beta|<r$ for some $r>1$, all $n \geq 1$, and all derivations of length $n$ in $G$. 
(iii) Consider $\beta \in V^{*}$ such that $|\beta|=r$ and a derivation $\Gamma_{0} \Rightarrow \ldots \Rightarrow \Gamma_{n}$ of length $n$ in $G$ such that $\beta$ is not a prefix of $\Gamma_{0}$ and $\beta$ is a prefix of $\Gamma_{n}$. Let $\left\{\left\langle\left(B_{i}, P_{i}, C_{i}\right),\left(B_{i}, Q_{i}, C_{i}\right)\right\rangle\right\}_{i=1}^{n}$ be a production sequence for this derivation. Let $\Gamma_{0}=Y_{1} \ldots Y_{t}, t \geq 1$, each $Y_{i} \varepsilon V_{0}$. If some step of $\Gamma_{0} \Rightarrow \ldots \Rightarrow \Gamma_{n}$ transforms $Y_{1}$, then the argument is just as in (i). Suppose no step of $\Gamma_{0} \Rightarrow \ldots \Rightarrow \Gamma_{n}$ transforms any part of $Y_{1} \ldots Y_{q-1}$ for some $q \leq t$ but some step transforms $Y_{q} q^{\cdot}$ Since $B$ is not a prefix of $\Gamma_{0}=Y_{1} \ldots Y_{t}$, this implies $q \leq|\beta|=r_{0}$ Since $Y_{Q}$ is transformed at some step, $Y_{Q} \varepsilon V-\Sigma_{0}$ Let $\Gamma_{k-1} \Rightarrow \Gamma_{k}$ be the first step such that $Y_{q}$ is transformed. Since no part of $Y_{I} \ldots Y_{q-1}$ is transformed in $\Gamma_{0} \Rightarrow \ldots \Rightarrow I_{n}$ and $Y \in V-\Sigma$, no part of $Y_{1} \ldots Y_{q}$ can serve as part of left context in any step of $\Gamma_{0} \Rightarrow \ldots \Rightarrow \Gamma_{k-1}$.

If the rule applied in $\Gamma_{k-1} \Rightarrow \Gamma_{k}$ is a context-free rule, then the argument is just as in (i). If the rule used in $\Gamma_{k-1} \Rightarrow \Gamma_{k}$ is a non-context-free rule, say $\alpha_{1} Y_{q} \alpha_{2} \rightarrow \alpha_{1} y \alpha_{2}$ then by choice of $q, \alpha_{I}$ must be a suffix of $Y_{I} \ldots Y_{q-I}$, and so $\left|\alpha_{1}\right| \leq q-1<q \leq|\beta|=r$. Thus $r_{k-1}=B_{k} P_{k} C_{k}$ where $\mathbb{P}_{k}=\alpha_{1} Y_{q} \alpha_{2}$ and $B_{k} \alpha_{1}=Y_{1} \ldots Y_{q-1}$. If $B_{k} \alpha_{1} Y_{q} \alpha_{2}$ is a prefiz of $\Gamma_{O}$, then the argument is just as in (i), applying $\alpha_{1} Y_{q} \alpha_{2} \rightarrow \alpha_{1} \gamma \alpha_{2}$ first and noticing that this step is $q \leq|\beta|<M(|B|)$ bounded. 
If $B_{k} \alpha_{1} Y_{q} \alpha_{2}$ is not a prefix of $\Gamma_{0}$, then by choice of $q$ the application of rules in $\Gamma_{0} \Rightarrow \ldots \rightarrow \Gamma_{k-1}$ transform no symbols in $\Gamma_{0}=Y_{1} \ldots Y_{\tau}$ to the left of $Y_{q+1}$ and these steps use no part of $B_{k} a_{1} Y_{q}=Y_{I} \ldots Y_{q}$ as context. Thus for each $i=I, \ldots, k-1, B_{k} \alpha_{1} Y_{q}=Y_{I} \ldots Y_{q}$ is a prefix of $B_{i}$ say $B_{i}=B_{k} \alpha_{1} Y_{q} D_{i}$ for some $D_{i} \in V^{*}$. If for some $i=1 \ldots k-1$, $\Delta_{i-1}=D_{1} P_{1} C_{i}$, and $\Delta_{k-1}=D_{k-1} Q_{k-1} C_{k-1}$, then $\Delta_{0} \Rightarrow \ldots \Rightarrow \Delta_{k-1}$ is a derivation of length $k-1$ in $G$ (with production sequence $\left.\left\{\left\langle\left(D_{i}, P_{i}, C_{i}\right),\left(D_{i}, Q_{i}, C_{i}\right)\right\rangle\right\}_{i=1}^{k-1}\right)$ such that $a_{2}$ is not a prefix of $\Delta_{0}$ bur $\alpha_{2}$ is a prefix of $\Delta_{k-1}$. Since $\alpha_{1} \mathrm{Yqa}_{2} \rightarrow \alpha_{1} \gamma \alpha_{2}$ is a rule in $k,\left|\alpha_{1}\right| \geq\left|\alpha_{2}\right|$. Also, $\left|\alpha_{1}\right|<r$. Hence, $\left|\alpha_{2}\right|<I$ and $k-1<n$ so that the induction hypothesis applies to the derivation $\Delta_{0} \Rightarrow \ldots \Rightarrow \Delta_{k-1}$ and the string $\alpha_{2}$, that is, there is a derivation $\nabla_{0} \Rightarrow \ldots \Rightarrow \nabla_{k-1}$ of length $k-1$ in $G$ such that $\nabla_{0}=\Delta_{0}, \nabla_{k-1}=\Delta_{k-1}$ and the step $\nabla_{0} \Rightarrow \nabla_{1}$ is $M\left(\left|\alpha_{2}\right|\right)$ bounded.

Construct the derivation $\pi_{0} \Rightarrow \ldots \Rightarrow \pi_{n}$ as follows. For each $i=1, \ldots, k-1$, let $\Pi_{i}=B_{k} \alpha_{1} Y_{q} \nabla_{i}$, so that $\Pi_{0} \Rightarrow \ldots \Rightarrow \Pi_{k-1}$ Now $\Pi_{0}=B_{k} \alpha_{1} Y_{q} \nabla_{0}=B_{k} \alpha_{1} Y_{q} \Delta_{0}=B_{k} \alpha_{1} Y_{q} D_{1} P_{1} C_{1}=B_{1} P_{1} C_{1}=\Gamma_{0}$ and $\Pi_{k-1}=B_{k} \alpha_{1} Y_{q} \nabla_{k-1}=B_{k} \alpha_{1} Y_{q} \Delta_{k-1}=B_{k} \alpha_{1} Y_{q} D_{k-1} Q_{k-1} C_{k-1}=$ $B_{k-1} Q_{k-1} C_{k-1}=\Gamma_{k-1}$. For $i=k, \ldots, n$, let $\Pi_{i}=\Gamma_{i}$. Thus, $\Gamma_{0}=\Pi_{0} \Rightarrow \ldots \Rightarrow \Pi_{k-1}=\Gamma_{k-1}$ and $r_{k}=\Pi_{k} \Rightarrow \ldots \Rightarrow \Pi_{n}=\Gamma_{n}$ so that $\Pi_{0} \Rightarrow \ldots \Rightarrow \Pi_{n}$. Further, the step $\Pi_{0} \Rightarrow \Pi_{1}$ is 
$\left|B_{k} \alpha_{1} Y_{q}\right|+M\left(\left|\alpha_{2}\right|\right)$ bounded since $\nabla_{0} \Rightarrow \nabla_{1}$ is $M\left(\left|\alpha_{2}\right|\right)$ bounded. But $\left|B_{k, 1} \alpha_{q}\right|=q \leq|\beta|$ and $\left|\alpha_{2}\right| \leq\left|\alpha_{1}\right|<|\beta|$ so that $\left|B_{k} \alpha_{1} Y_{q}\right|+M\left(\left|\alpha_{2}\right|\right) \leq M(|\beta|)$. Thus $\pi_{0} \Rightarrow \pi_{1}$ is $M(|\beta|)$ bounded. $\square$

Lemana 2. Let $G=(V, \Sigma, R, X)$ be a Type 0 grammar which satisfies the hypothesis of Theorem 1 . For any $w \in I(G)$, if $x \Rightarrow \Gamma_{1} \Rightarrow \ldots \Rightarrow \Gamma_{n}=$ is a derivation in $G$ such that there is a least $t$ where the step $I_{t} \Rightarrow \Gamma_{t+1}$ is not $M_{G}$ bounded, then there exist $\pi_{1}, \ldots, \pi_{n} \varepsilon V^{*}$ such that $x \Rightarrow \pi_{I} \Rightarrow \ldots \Rightarrow \pi_{n}$ is a derivation in $G, \pi_{n}=w$, and the derivation $x \Rightarrow \Pi_{I} \Rightarrow \ldots \Rightarrow \Pi_{t+I}$ is $M_{G}$ bounded.

Proof. Let $\Gamma_{t}=a_{1} \ldots a_{p} z_{I} \ldots z_{q}$ where each $a_{i} \varepsilon \Sigma$, each $z_{j} \varepsilon V$, and $z_{1} \varepsilon V-\Sigma$. Since the step $\Gamma_{t} \Rightarrow \Gamma_{t+1}$ is not $M_{G}$ bounded, $z_{2} \ldots z_{\mathrm{q}} \neq e_{\text {e. Since }} \mathrm{x} \stackrel{*}{\Rightarrow} \Gamma_{\mathrm{t}} \stackrel{*}{\Rightarrow} \Gamma_{\mathrm{n}}$ and $\Gamma_{\mathrm{n}}=\mathrm{w} \varepsilon \Sigma^{*}$, there is some first step of $\Gamma_{t} \Rightarrow \ldots \Rightarrow \Gamma_{n}$ which transforms $z_{1}$. Let $\Gamma_{j} \Rightarrow \Gamma_{j+1}$ be that step, so that $t<j$. Since $z_{1} \varepsilon V-\Sigma$, no part of $a_{1} \ldots a_{p} z_{1}$ serves as part of the left context of any rule applied in $\Gamma_{t} \Rightarrow \ldots \Rightarrow \Gamma_{j}$ " Since $z_{1}$ is the leftmost nonterminal symbol in each of $\Gamma_{t}, \Gamma_{t+1}, \ldots, \Gamma_{j}$, no part of $a_{1} \ldots a_{p} z_{1}$ serves as part of the right context of any rule applied in $\Gamma_{t} \Rightarrow \ldots \Rightarrow \Gamma_{j}$. Thus, as in the proof of Lemma 1 , the derivation $\mathrm{X} \Rightarrow \pi_{1} \Rightarrow \ldots \Rightarrow \pi_{n}$ can be constructed by rearranging the order of application of rules in the derivation $\Gamma_{t} \Rightarrow \ldots \Rightarrow \Gamma_{j}$. 
In particular, if the rule applied in $\Gamma_{j} \Rightarrow \Gamma_{j+1}$ is a context-free rule $z_{1} \rightarrow \gamma$ or is a context-sensitive rule $\alpha z_{1} \beta \rightarrow \alpha \gamma \beta$ and $\Gamma_{t}=$

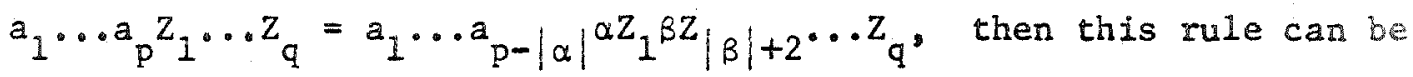
applied to $\pi_{t}$ to yield $\pi_{t+1}=a_{1} \ldots a_{p} \gamma z_{2} \ldots z_{q}$, where $\Pi_{i}=I_{i}$ for $i=1, \ldots, t, j+1, \ldots, n$, and $\Pi_{t+2}, \ldots, \Pi_{j+1}$ are obtained from $\Gamma_{t} \Rightarrow \ldots \Rightarrow \Gamma_{j}$ just as in the proof of Lemma 1 . In this case the step $\Pi_{t} \Rightarrow \Pi_{t+1}$ is 1 -bounded.

If the rule applied in $\Gamma_{j} \Rightarrow \Gamma_{j+1}$ is a context-sensitive rule $\alpha z_{1} \beta \rightarrow \alpha \gamma \beta$ and $\alpha z_{1} \beta$ is not a prefix of $\Gamma_{t}$, then $\beta$ is not a prefix of $Z_{2} \cdots z_{q}$ but is a prefix of $\delta$ where $\Gamma_{j}=\alpha z_{1} \delta$. Since the miles applied in $\Gamma_{t} \Rightarrow \ldots \Rightarrow \Gamma_{j}$ use no part of $a_{1} \ldots a_{p} z_{1}$ as either left or right context, this sequence of rules can be applied to $\mathrm{Z}_{2} \cdots \mathrm{Z}_{\mathrm{q}}$ to obtain $\delta$ in a derivation of length $j-t$. By Lemma $I$ this derivation can be converted to another derivation $\mathrm{z}_{2} \ldots \mathrm{z}_{\mathrm{q}} \Rightarrow \ldots \Rightarrow \delta$ of length $\mathrm{j}$ - $\mathrm{c}$ such that the first step is $M(\beta)$ bounded. From the latter derivation (just as in the proof of Lemma 1$), \pi_{\tau} \Rightarrow \ldots \Rightarrow \pi_{j}$ is obtained such that $\Pi_{t}=\Gamma_{t}, I_{j}=\Gamma_{j}$ and the step $\Pi_{t} \Rightarrow \Pi_{t+1}$ is $M(\beta)+1 \leq M_{G}$ bounded. Letting $\Pi_{i}=\Gamma_{i}$ for $i=1, \ldots, t-1, j+1, \ldots, \pi_{\text {, }}$ one obtains detivation $x \Rightarrow n_{1} \Rightarrow \ldots \Rightarrow \pi_{n}=\Gamma_{n}=w$ where the derivation $x \Rightarrow n_{1} \Rightarrow \ldots \Rightarrow n_{t+1}$ is ${ }^{M}$ bounded. 


\section{Proof of Theorem 1.}

For any $w \varepsilon \bar{L}(G)$, consider any derivation $x \Rightarrow \Gamma_{1} \Rightarrow \ldots=\Gamma_{\mathrm{n}}=\mathrm{w}$ in $G$. Either this derivation is $M_{G}$ bounded, or applying Lema 2 at most $n-2$ times yields a derivation $x \Rightarrow \pi_{1} \Rightarrow \ldots \Rightarrow \pi_{n}=w$ in $G$ which is $\mathbb{M}_{G}$ bounded. Hence $w \in \operatorname{LEFT}\left(M_{G}, G\right)$. Thus $L(G) \subseteq \operatorname{LEFT}\left(M_{G}, G\right) \subseteq$ $L(G)$. Since $\operatorname{LEFT}\left(M_{G}, G\right)$ is context-free, $L(G)$ is context-free.

Let $G=(V, \Sigma, R, X)$ be a Type 0 grammar which satisfies the hypothesis of Theorem 1, so that if $\alpha Z B \rightarrow$ ay $\varepsilon R_{0}$ then $|\alpha| \geq|B|$. If this restriction on length is weakened, then $L(G)$ is not necessarily contextfree. To see that this is true, note that there exist Type 0 grammars $G=(V, \Sigma, R, X)$ such that each non-context-free rule is of the form $Z Y \rightarrow Z^{\prime} Y$ where $Z \varepsilon V-\Sigma$, and $Z^{\prime}, Y \varepsilon V$, and such that $L(G)$ is not context-free? With no loss of generality, assume that in such a gramar $G$, each context-free rule is of the form $z \rightarrow \gamma$ where $|\gamma| \leq 2$. Let be a new symbol not in $v_{v} \Sigma_{1}=\Sigma U\{c\}$, and $v_{1}=v U\{c\}$. Let

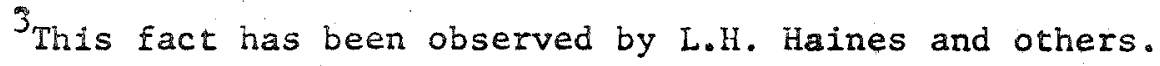




$$
\begin{aligned}
R_{1}= & \{Z \rightarrow e \mid Z \rightarrow e \varepsilon R\} \cup\{Z \rightarrow Y \mid Z, Y \varepsilon V, Z \rightarrow Y \varepsilon R\} U \\
& \left\{Z \rightarrow c Y_{1} c Y_{2} c, Z \rightarrow c Y_{1} c Y_{2}, Z \rightarrow c Y_{1} Y_{2}, Z \rightarrow c Y_{1} Y_{2} c\right. \\
& \left.Z \rightarrow Y_{1} c Y_{2} c, Z \rightarrow Y_{1} Y_{2} c, Z \rightarrow Y_{1} c Y_{2} \mid Z, Y_{1}, Y_{2} \varepsilon V, Z \rightarrow Y_{1} Y_{2} \varepsilon R\right\} U \\
& \left\{c Z c Y \rightarrow c Z^{\prime} c Y \mid Z, Y, Z^{\prime} \varepsilon V, Z Y \rightarrow Z^{\prime} Y \in R\right\} .
\end{aligned}
$$

The grammar $G_{1}=\left(V_{1}, \Sigma_{1}, R_{1}, X\right)$ is such that each non-context-free rule is of the form $\alpha Z \beta \rightarrow \alpha \gamma \beta$ where $\alpha \varepsilon \Sigma_{1}$ and $1=|\alpha|<|\beta|=2$. If $h: \Sigma_{1}^{*} \rightarrow \Sigma^{*}$ is the homomorphism determined by defining $h(c)=e$ and for every $\alpha \varepsilon \Sigma, h(a)=a$, then clearly $h\left(L\left(G_{1}\right)\right)=L(G)$. Since $I(G)$ is not context-free, $L\left(G_{1}\right)$ is not context-free. Hence, the length restriction cannot be weakened.

It should be noted that the theory of phrase-structure gramars or rewriting systems is symmetric with respect to left and right. Hence, if the restriction on the form of the rules in Theorem $I$ is altered to $\alpha Z \beta \rightarrow \alpha \gamma \beta$ where $\beta \varepsilon \Sigma^{*}, Z \varepsilon V-\Sigma, \alpha, \gamma \varepsilon V^{*}$, and $|\alpha| \leq|\beta|$, then again $L(G)$ is context-free. 
Section 3.

This section is devoted to showing that if the rules of a grammar have only terminal strings as context, then the language generated is context-free. Formally, this is stated in the following theorem.

Theorem 2. If $G=(V, \Sigma, R, X)$ is a Type 0 gramar such that every noncontext-free rule is of the form $\alpha Z \beta \rightarrow \alpha \gamma \beta$ where $\alpha \varepsilon \Sigma^{*}$ and $\beta \varepsilon \varepsilon^{*}$, $Z \in V-\Sigma$, then $L(G)$ is context-free.

Intuitively one sees that "messages" cannot be transmitted over sufficiently long terminal strings and here it is only terminal strings which are allowed as context. However, the formal proof of the theorem is based on a lemma which allows one to reduce the length of terminal context, so that by repeated use a context-free grammar is generated.

Notation. For any Type 0 grammer $G=(V, \Sigma, R, X)$, let $L_{G}=\max \{|\alpha|$ $\alpha Z \beta \rightarrow \alpha \gamma \beta \varepsilon R\}$ and $R_{G}=\max \{|\beta| \mid \alpha Z \beta \rightarrow \alpha \gamma \beta \varepsilon R\}$.

Lemma 3. Let $G=(V, \Sigma, R, X)$ be a Type 0 grammar satisfying the hypothes is of Theorem 2. If $R_{G} \geq L_{G}$ and $R_{G} \geq 1$, then one can construct a Type 0 grammar $G_{1}$, a regular set $T$, and a gsm $f$ such that

(i) $G_{1}$ satisfies the hypothesis of Theorem 2 .

(ii) $R_{G_{1}}=R_{G}-1$ and $L_{G_{1}}=I_{G}$, and (iii) $f\left(L\left(G_{1}\right) \cap T\right)=L(G)$.

\footnotetext{
${ }^{4}$ See [3] for definitions and facts about regular sets and gsm's.
} 


\section{Proof of Theorem 2 .}

The theory of phrase-structure grammars is symetric with respect to left and right, so that Lemma 3 still holds if $R_{G}$ and $L_{G}$ (and $R_{G_{1}}$ and $L_{G_{I}}$ ) are intercianged throughout--refer to the result as Lema $3^{4}$. Given $G$ as in the hypothesis, appiying Lemmas 3 and $3^{\circ} \mathrm{m}=\mathrm{R}_{G}+L_{G}$ times yields a sequence $G_{1}, \ldots, G_{m}$ of gramars, a sequence $\mathrm{T}_{1}, \ldots, \mathrm{I}_{\mathrm{m}}$ of regular sets, and a sequence $f_{1}, \ldots, f_{m}$ of $\mathrm{gsm}^{\prime} \mathrm{s}$ such that for $i=1, \ldots, m_{0} f_{i}\left(L\left(G_{i}\right) \cap T_{i}\right)=L\left(G_{i-i}\right)$, where $G_{0}=G$ and $G_{m}$ is contextfree [since $L_{G_{m}}=R_{G_{m}}=0$ implies $G_{m}$ is context-free]. Since the family of context-free languages is closed under intersection with regulat sets and under gsm mappings, this implies that $L(G)=L\left(G_{0}\right)$ is contextfree.

The proof of Lema 3 rests on the following observation. If $\mathrm{X} \Rightarrow \Gamma_{1} \Rightarrow \ldots \Rightarrow \Gamma_{\mathrm{n}}$ is any derivation in $\mathrm{G}$ and some $\Gamma_{i}$ has a terminal substring of length $R_{G}$ of greater, then every step of $r_{i} \Rightarrow \ldots \Rightarrow \Gamma_{D}$ either transforms a symbol to the right of $\beta$ independent of what is to the left of $B$ or transforms a symbol to the left of $\beta$ independent of what is to the right of $\beta$. 


\section{Proof of Lemma 3.}

Without loss of generality, assume that every non-context-free rule in $R$ either has left context but no right context, or has right context but no left context. Partition the set $R$ as follows: let

$$
\begin{aligned}
& S_{1}=\{Z \rightarrow \gamma \varepsilon R \mid Z \varepsilon V-\Sigma\}, \\
& S_{2}=\left\{\alpha Z \rightarrow \alpha \gamma \varepsilon R \mid \alpha \varepsilon \Sigma^{*}, Z \varepsilon V-\Sigma \text {, and }|\alpha|<R_{G}\right\} \text {, } \\
& S_{3}=\left\{\alpha Z \rightarrow \alpha \gamma \varepsilon R \mid \alpha \varepsilon \Sigma^{*}, Z \varepsilon V-\Sigma \text {, and }|\alpha|=R_{G}\right\}, \\
& S_{4}=\left\{Z \beta \rightarrow \gamma \beta \varepsilon R \mid Z \varepsilon V-\Sigma, B \varepsilon \Sigma^{*} \text {, and }|\beta|<R_{G}\right\} \text {, and } \\
& S_{5}=\left\{Z B \rightarrow \gamma \beta \varepsilon R \mid Z \varepsilon V-\Sigma, \beta \varepsilon \Sigma^{*} \text {, and }|\beta|=R_{G}\right\} \text {. }
\end{aligned}
$$

For each $\beta \in \Sigma^{*}$ such that $|\beta|=R_{G}$ and there exist $z \varepsilon V-\Sigma, \quad y \varepsilon V^{*}$ with $Z \beta \rightarrow \gamma \beta \varepsilon S_{5}$, let [B] be a new symbol, and let $\Sigma_{1}$ be the set of such new symbols. For each such $\beta$, let $\beta_{-1}$ be the prefix of $\beta$ of length $|\beta|-1$, i.e., if $\beta=\delta$ a where a $\varepsilon \Sigma$, then $\beta_{-1}=\delta$; since $|B|=R_{G},\left|B_{-I}\right|=R_{G}-1$.

Construct the grammar $G_{1}=\left(V_{1}, \Sigma \cup \Sigma_{1}, R_{1}, X\right)$ as follows. Let $V_{1}=V \cup \Sigma_{1}$ Let $U_{5}=\left\{Z \beta_{-1} \rightarrow \gamma \beta_{-1}[\beta] \beta \beta_{-1} \mid Z \varepsilon V-\Sigma\right.$ and $\left.Z \beta \rightarrow \gamma \beta \varepsilon S_{5}\right\}$. Let $R_{1}=\left(R-S_{5}\right) \cup U_{5}$. From the construction it is clear that $G_{1}$ is a Type 0 grammar such that $L_{G_{I}}=L_{G}$ and $R_{G_{1}}=R_{G}-1$. 


$$
\text { Let } T=\left(\Sigma \cup\left(\underset{[\beta] \varepsilon \Sigma_{1}}{\bigcup}\left(\beta_{-1}[\beta]\right)^{*} \beta_{-1}[\beta] \beta\right)\right)^{*} \text { so that } T \text { is a regular }
$$

set. Let $f$ be a gsm which yields the identity mapping on $\sum$ until

a symbol $[\beta] \in \Sigma_{1}$ is scanned. Strings of the form $[B] \beta_{-1}$ are etased and $\bar{f}$ returns to its initial mode of operation. Any other operation outputs "garbage". Hence for any $[\beta] \varepsilon \Sigma_{1}$ and any $n \geq 0$, $E\left(\beta_{-1}\left([\beta] \beta_{-1}\right)^{n}[\beta] \beta\right)=\beta_{-I} f\left(\left([\beta] \beta_{-1}\right)^{n+1} a\right)=\beta_{-I} a=\beta$ where a $\varepsilon \sum$ and $B=\beta_{-1} a$. Also, $f(w)=w$ if and only if $w \in \Sigma^{*}$. Before proving that $f\left(L\left(G_{1}\right) \cap T\right)=L(G)$, let us informally explain the construction of $G_{1}$ and the role of $T$ and $f$. Since $R-S_{5}=$ $R_{1}=U_{5}$ and $U_{5}$ is a "copy" of $S_{5}$, it is enough to explain the use of rules in $U_{5}$. When a rule $Z \beta_{-1} \rightarrow \gamma_{-1}[\beta] \beta_{-1}$ is applied to a string $E Z\left(\beta_{-1}[\beta]\right)^{t} \dot{\beta}_{-1} F$ to obtain $E Y\left(\beta_{-k}[\beta]\right)^{t+1} \beta_{-1} F$, one "guesses" that $\beta$ is a prefix of $\beta_{-1} F$ so that an application of $Z \beta \rightarrow \gamma \beta$ in $G$ is being imitated. The new occurrence of the symbol [B] serves as a "marker" to fndicate this guess and also as a "barrier" so that further steps take place either to the right of $\left(\beta_{-1}[B]\right)^{t+1}$ or to the left of $\left([B] B_{-1}\right)^{t+1}$. The "new" copy of $\beta_{-1}$ in $\gamma \beta_{-1}[\beta] \beta_{-1}$ is availabie for use as riter context in the future application of some rule. The "old" $\beta-1$ can still serve as part of left context, since if $\beta=\beta_{-1}{ }^{a}$, one still wishes to be able to apply a rule such as $\delta a Y \rightarrow \delta a \Psi$ if such a rule is in $R$. By hypothesis $I_{G} \leq R_{G}$, so that $|\delta a| \leq|B|=R_{G}$. Hence if one were able to apply this rule in a derivation in $G$ and if the guess that $B$ is present is correct. 
then one can still apply this rule in an "imitating" derivation in $G_{1}-$ the symbol $[\beta]$ does not cause a conflict since $|[\beta] \beta-1|=1+\left|\beta_{-1} a\right|=$ $1+|\beta|=1+R_{G}>I_{G} \cdot$

The reguiar set $T$ serves as a "filter" to restrict attention to terminal strings which do have substrings in $\left(\beta_{-1}[\beta]\right)^{\tau+1} \beta-i_{0} e_{0}$ to check that the guesses were correct. The symbol [B] also serves as a marker to tell the gsm to erase the substring $[B] B_{-1}$.

The equality $f\left(L\left(G_{1}\right) \cap T\right)=L(G)$ is established by showing that a derivation in $G$ resulting in a string in $L(G)$ can be imitated by a derivation in $G_{1}$ resulting in a string in $L\left(G_{1}\right) \cap T$, with the role of $f$ being obvious. The inclusion $f\left(L\left(G_{I}\right) \cap T\right) \subseteq L(G)$ is somewhat more complicated only because a derivation in $G_{1}$ resulting in a string lin $L\left(G_{1}\right) \cap T$ may need to be "re-arranged" in order to be imitated in $G-$. the "guess" may have been made too soon. The proofs of these inciusions are only sketched since the detailed induction arguments do not yield any additional insight.

Claim I. $L(G) \subseteq E\left(L\left(G_{1}\right) \cap T\right)$

sketch of the proof. It is sufficient to show that derivations in 6 can be imitated in $G_{1}$ such that any resulting terminal string is also in $T$. Derivations in $G$ which do not use rules in $S_{5}$ can be considered to be derivations in $G_{1}$ and $L(G) \subseteq \Sigma^{*} \subset T$. Thus one need be concerned only with those derivations in $G$ which do use rules in $S_{5}$. 
The set $R_{1}$ of rules of $G_{1}$ contains all the rules in $R-S_{5}$ as well as the set $U_{5}$, which is a "copy" of $S_{5^{\circ}}$ A rule in $U_{5}$ is of the form $Z \beta_{-1} \rightarrow \alpha \beta_{-1}[\beta] \beta_{-1}$ where $Z \in V-\Sigma, \beta_{-1} \varepsilon \Sigma^{*},\left|\beta_{-1}\right|=R_{G}-I_{*}$ and $2 \beta \rightarrow \gamma \beta$ is in $S_{5}$. Hence the symbol $[B]$ is generated only as part of a string $\beta_{-1}[\beta] \beta_{-1}$. If one "imitates" a derivation of $G$ in $G_{1}$, then to imitate an application of $Z \beta \rightarrow \gamma \beta$, the rule $Z \beta_{-1} \rightarrow \gamma \beta-1[\beta] \beta-1$ in $U_{5}$

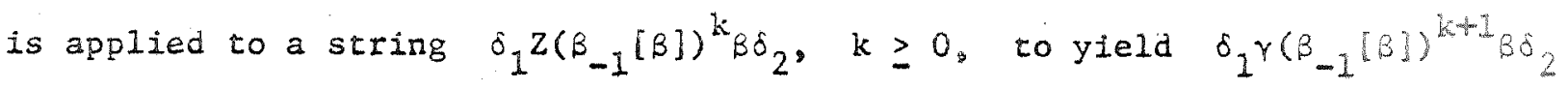
so that $[\beta]$ is generated as part of $\left(\beta_{-I}[\beta]\right)^{k+1} \beta$. Thus it is easy to see that if $x \Rightarrow \Gamma_{I} \Rightarrow \ldots \rightarrow \Gamma_{n}$ is a derivation in $G$ with $\Gamma_{n} \varepsilon \Sigma^{*}$ then one can construct a derivation $x=\Pi_{I} \Rightarrow \ldots \Rightarrow \Pi_{n}$ in $G_{1}$ with $\pi_{n} \varepsilon\left(\Sigma \cup \Sigma_{I}\right)^{*}$ such that $\pi_{n} \in T$ and $f\left(\pi_{n}\right)=\Gamma_{n}$. Hence, $L(G) \subseteq$ $f\left(L\left(G_{1}\right) \cap T\right) \cdot \quad \square$

Claim 2. $f\left(L\left(G_{1}\right) \cap T\right) \subseteq L(G)$.

Sketch of the proof. It is sufficient to show that derivations in $G_{2}$ which generate strings in $L\left(G_{1}\right) \cap T$ can be imitated in $G$. Such derivations in $G_{1}$ generate strings in $L\left(G_{1}\right) \cap \Sigma^{*}$ if and only if they use no rules from $U_{5}$. But clearly such derivations are already derivations in $G_{3}$ so $L\left(G_{1}\right) \cap \Sigma^{*} \subseteq L(G) ;$ also, $f\left(L\left(G_{1}\right) \cap \Sigma^{*}\right)=L\left(G_{1}\right) \cap \Sigma^{*}$. Hence one need consider only those derivations in $G_{I}$ which use at least one application of a rule in $\mathrm{U}_{5}$. 
Suppose $x \Rightarrow \Gamma_{1} \Rightarrow \ldots \Rightarrow \Gamma_{n}$ is a derivation in $G_{1}$ such that $\Gamma_{n} \varepsilon L\left(G_{1}\right) \cap T$ and such that for some $k \leq n$ the rule applied at the step $\Gamma_{k-1} \Rightarrow \Gamma_{k}$ is a rule in $U_{5}$. Let $\left\{\left\langle\left(B_{i}, P_{i}, C_{i}\right),\left(B_{i}, Q_{i}, C_{i}\right)\right\rangle\right\}_{1=1}^{I n}$ be a production sequence for $x \rightarrow \Gamma_{1} \rightarrow \ldots \Rightarrow \Gamma_{n}$, and let $P_{k} \rightarrow Q_{k}$ be $2 \beta_{-1} \rightarrow \gamma \beta_{-1}[\beta] \beta_{-1}$, where $Z \varepsilon V-\Sigma$ and $\beta_{-1} \varepsilon \Sigma^{*}$. If $P_{k} C_{k}=Z\left(\beta_{-1}[\beta]\right)^{t} \beta D$ for some $t \geq 0$ and $D \varepsilon V_{1}^{*}$, then in the "imitating" derivation $x \Rightarrow \pi_{1} \Rightarrow \ldots \Rightarrow \pi_{n}$ in $G$, the rule $Z B \rightarrow \gamma \beta$ can be applied at the step $\pi_{k-1} \Rightarrow \pi_{k}$. Since $f((\beta-1[B])+1 \beta)=$ $\beta$, the substring $\left(\beta_{-1}[B]\right)^{t+1} \beta$ of $\Gamma_{k}$ is mapped onto the appropriate substring $B$ of $\Pi_{k}$.

Suppose for some $t \geq 0, Z\left(\beta_{-1}[B]\right)^{t} \beta_{-1}$ is a prefix of $p_{k} C_{k}$ but for every $m \geq 0, \quad Z\left(\beta_{-1}[\beta]\right)^{m_{B}}$ is not a prefix of $P_{k} C_{k}$. Let $\Gamma_{q-1} \rightarrow \Gamma_{q}$ be the first step which generates an accurrence of [B]. Since no rule of $R_{1}$ has any symbol of $\Sigma_{1}$ on its lefthand side and since $\left|B_{-1}\right|=R_{G_{1}}$. one loses no generality by assuming that for some $m, q<m \leq n$ every step of $\Gamma_{\mathrm{G}} \Rightarrow \ldots \rightarrow \Gamma_{\mathrm{m}}$ transforms a symbol to the left of $[\beta]$ and every step of $\Gamma_{\mathrm{m}} \Rightarrow \ldots \Leftrightarrow \Gamma_{\mathrm{n}}$ transforms a symbol to the right of $[\beta]$. Since $\Gamma_{\mathrm{n}} \in \mathrm{T}$, the derivation $\Gamma_{\mathrm{m}} \Rightarrow \ldots \Rightarrow \Gamma_{\mathrm{n}}$ produces a substring [8]B. Hence in the imitating derivation $x \Rightarrow \pi_{I} \Rightarrow \ldots \Rightarrow \pi_{n}$ in $G$, the portion $\pi_{q-1} \Rightarrow \ldots \Rightarrow \pi_{q-1+n-m}$ imitates $\Gamma_{m} \Rightarrow \ldots \Rightarrow \Gamma_{n}$ so that when imitating $\Gamma_{q-1} \Rightarrow \Gamma_{q}$, the string $\beta$ is available to use as right context. This step is imitared by $\pi_{q-I+n-m} \Rightarrow \dot{I}_{q+n-m}$ and then $\Gamma_{q} \rightarrow \ldots: \Gamma_{m}$ is imitated by $\pi_{q+n-m} \Rightarrow \ldots \Rightarrow \pi_{n}$. 
A construction similar to that in the proof of Lema 3 can be used in conjunction with the result of Ginsburg and Greibach cited in Section 1 to establish the following generalization of Theorem 2.

Corollary. If $G=(V, \Sigma, R, X)$ is a gramar such that every non-contextfree rule is of one of the forms

(i) $\alpha \rho \rightarrow \alpha \theta$ where $\alpha \varepsilon \Sigma^{*},|\alpha| \geq 1$, and $\rho \varepsilon(V-\Sigma)^{*}$,

(ii) $O B \rightarrow O B$ where $\beta \varepsilon \Sigma^{*},|\beta| \geq 1$, and $\rho \varepsilon(V-\Sigma)^{*}$,

then $L(G)$ is context-free.

There is one further restriction which generalizes the hypothesis of both Theorems 1 and 2 , that is, let $G=(V, \Sigma, R, X)$ be a Type $O$ grammat such that every non-context-free rule is of one of the forms
(i) $\alpha Z B \rightarrow \alpha \gamma \beta$ where $\alpha \varepsilon \Sigma^{*}, Z \varepsilon V-\Sigma, \beta, \gamma \in V^{*}$, and $|\alpha| \geq|B| *$
(ii) $\alpha z \beta \rightarrow \alpha \gamma \beta$ where $\beta \varepsilon \Sigma^{*}, Z \in V-\Sigma, \alpha, \gamma \varepsilon V^{*}$, and $|\alpha| \leq|\beta|$

Thus it is required that either left or right context be a terminal scring and in either case, the terminal context has length at least as great as the other context. We conjecture that this restricton forces the language generated to be context-free. 
1. R. Book, Time-bounded grammars and their languages, J. Computer and System Sciences 5 (1971), to appear.

2. R. Evey, The Theory and Application of Pushdown Store Machines, Ph.D. Dissertation Harvard Univ., 1903. Also appears as Math. Linguistics and Automatic Translation NSF-10 (1963), Computation Lab., Harvard Univ., Cambridge, Mass.

3. S. Ginsburg, The Mathematical Theory of Context-free Languages McGratHill, 1966.

4. S. Ginsburg and S.A. Greibach, Mappings which preserve context-sensitive languages, Information and Control 9 (1966), 563-582.

5. A. Gladkit, on the complexity of dexivations in phrase structure gramimars, Algebri i Logika Sem. 3 (1964), 29-44.

6. T. Griffiths, Some remarks on derivations in general rewriting systems, Information and Control 12 (1968), 27-54.

7. T. Hibbard, Scan Limited Automata and Context Limited Grammars, Ph,D. Dissertation, Univ, of California, Los Angeles, 1966.

8. G.H. Matthews, Discontinuity and asymetry in phrase structure gramars. Information and Control 6 (1963), 137-146.

9. G.H. Matthews, A note on asymetry in phrase structure grammars. Information and Control 7 (1964), 360-365.

10. G.H. Matthews, Two-way languages, Information and Control 10 (1967), 111-119.

11. A. Salomaa, Formal Languages, in preparation. 